\title{
In vitro investigation of the mechanism underlying the effect of ginsenoside on the proliferation and differentiation of neural stem cells subjected to oxygen-glucose deprivation/reperfusion
}

\author{
JIAN GAO ${ }^{1}$, HUAJING BAI $^{1}$, QIANG LI $^{2}$, JIAN LI $^{3}$, FENG WAN $^{1}$, MO TIAN $^{1}$, \\ YUANYUAN LI ${ }^{1}$, YILUN SONG ${ }^{4}$, JIANPING ZHANG ${ }^{5}$ and YINCHU SI ${ }^{1}$ \\ ${ }^{1}$ Department of Anatomy, School of Basic Medical Sciences, ${ }^{2}$ School of Chinese Materia Medica, \\ ${ }^{3}$ School of Basic Medical Sciences, ${ }^{4}$ International School, Beijing University of Chinese Medicine, Beijing 100029; \\ ${ }^{5}$ Department of Anatomy, Zhejiang Chinese Medicine University, Hangzhou, Zhejiang 310053, P.R. China
}

Received April 4, 2017; Accepted November 1, 2017

DOI: $10.3892 / \mathrm{ijmm} .2017 .3253$

\begin{abstract}
The present study comprised a series of experiments to investigate the mechanism underlying the effect of ginsenoside on the self-renewal, proliferation and differentiation of neural stem cells (NSCs) undergoing oxygen-glucose deprivation/reperfusion $(\mathrm{OGD} / \mathrm{R})$ in vitro. The NSCs, which were isolated from the hippocampus of embryonic day 17 embryo rats, were subjected to OGD/R to establish an in vitro model of brain ischemia-reperfusion, following which different doses of ginsenoside were administered to the model. The proliferation of the NSCs was determined using MTT colorimetry and nestin/bromodeoxyuridine (BrdU) immunofluorescent doublelabeling. The NSCs were identified by measuring the expression of nestin, and the differentiation of NSCs was assessed through the immunofluorescent double-labeling of nestin/vimentin and nestin/neuron-specific class III $\beta$-tubulin (tuj-1). The protein levels of vascular endothelial growth factor (VEGF) and hypoxia-inducible factor- $1 \alpha$ (HIF-1 $\alpha$ ) were detected to investigate the function and mechanism of ginsenoside on ischemic stroke using an enzyme-linked immunosorbent assay. Marked increases in the optical density, area density and numbers of nestin/BrdU-, nestin/vimentin- and nestin/tuj-1-positive cells were found in the ginsenoside-treated group. Compared with the control group, enhanced expression levels of BrdU, tuj-1 and vimentin were found in the ginsenoside-treated group, suggesting that ginsenoside may significantly promote the proliferation and differentiation of NSCs. The results of the present study also showed that ginsenoside significantly
\end{abstract}

Correspondence to: Dr Yinchu Si, Department of Anatomy, School of Basic Medical Sciences, Beijing University of Chinese Medicine, Beijing 100029, P.R. China

E-mail: bjsiyinchu@vip.sina.com

Key words: ginsenoside, neural stem cells, hypoxia-inducible factor-1 $\alpha$, vascular endothelial growth factor, proliferation, differentiation increased the protein level of HIF-1 $1 \alpha(\mathrm{P}<0.05)$ in the NSCs exposed to $\mathrm{OGD} / \mathrm{R}$. These results indicated that ginsenoside may maintain NSC replication, promote NSC proliferation and promote NSC differentiation into neurons and astrocytes. Ginsenoside may initiate the expression of downstream VEGF, which is involved in promoting the survival, self-renewal and differentiation of NSCs.

\section{Introduction}

With a complex pathophysiology, ischemic stroke is damaging disease posing a significant threat to quality of life, and is the second leading cause of mortality and disability worldwide (1). As a contributor to mortality rates and loss of central nerve cells in the infarct region, ischemic cerebral artery thrombosis and embolus-induced ischemic cerebrovascular occlusion are encountered most frequently in cases of ischemic stroke (2). On the occurrence of initial ischemic injury, swelling of the damaged region of the brain may lead to secondary damage. The primary cause of neurological deficits is the neuronal damage caused by cerebral ischemia/reperfusion injury. Therefore, in cases of cerebral ischemia injury, the promotion of neuranagenesis is important as a measure of recovery of cerebral function. At present, despite numerous investigations into the pathological mechanism of ischemic stroke, few effective therapies are available for patients with the disease.

It has been found that cerebral ischemia can limit the supply of glucose and oxygen to brain tissue, which is considered to be a causal factor for neuron degeneration, the onset of ischemic stroke and Alzheimer's-type changes in the aged brain (3-5). As a hypoxic environment can have a detrimental effect on cell survival, hypoxic-ischemic cerebral injury can be utilized as a model for acquired neurodegenerative conditions with extensive parenchymal involvement. In practice, the model of oxygen-glucose deprivation (OGD) is used for investigations of cerebral ischemia injury in vitro, as its conditions can be easily controlled (6). In in vitro investigations, glucose-free culture medium is used for experiments involving hypoxic conditions, with an oxygen content of $<1 \%$, to simulate the status of cerebral ischemia and hypoxia caused by a 
short supply of oxygen and glucose. Reperfusion, comprising the resupplying of oxygen and glucose, is used to simulate the state of blood flow recovery in brain tissue. The OGD/reperfusion $(\mathrm{OGD} / \mathrm{R})$ model has been considered an ideal model for use in in vitro investigations of ischemia/reperfusion injury, as the conditions of the model can be easily controlled.

Neural stem cells (NSCs) are self-renewing multipotent cells, which can generate neurons, astrocytes and oligodendrocytes. The NSCs exist in the subventricular zone (SVZ) of the lateral ventricle and the dentate gyrus subgranular zone of the hippocampus throughout life $(7,8)$. The NSCs have neuroprotective functions due to their anti-inflammatory, glial scar-inhibitory, and anti-apoptotic effects; accordingly, they promote the recovery of patients with neurological disease (9). These characteristics of NSCs show that the NSCs can affect the regeneration of damaged brain tissues and possess therapeutic potential, which is promising for the treatment of pathological processes of disorders or injuries of the central nervous system. Although the characteristics of NSCs have been investigated extensively for over a decade (10), to enable the use of NSCs for accurate and safe therapies, key issues remain to be resolved, including the effective promotion, proliferation and induction of complete differentiation into neurons, of the NSCs.

It has been found that vascular endothelial growth factor (VEGF) is an important signaling molecule in angiogenesis and neurogenesis (11). Previous studies have shown that VEGF mRNA can be expressed in the ischemic cortex and hippocampus of rats with transient global cerebral ischemia, and the mechanism may be associated with the expression of hypoxia-inducible factor-1 $\alpha$ (HIF-1 $\alpha$ ) by cerebral injury and the regulatory downstream gene expression of $\operatorname{VEGF}(12,13)$. The mRNA expression of VEGF can promote the migration and proliferation of microvascular endothelial cells in the damaged region to form new blood vessels, improve blood supply and reduce cerebral ischemic injury; the regulatory downstream gene expression of VEGF can exert effects on neurotrophic activities to induce the proliferation and differentiation of NSCs and repair brain damage (14-17). Therefore, the HIF-1 $\alpha$-VEGF pathway may be a component of the pathogenetic mechanism of cerebral ischemic injury.

Ginsenoside is the major active component of ginseng, and it has been shown to be safe and effective in the treatment of acute ischemic stroke $(18,19)$. It has been reported that ginsenoside has effects on improving neurological outcome, decreasingthe infarct area, ameliorating mitochondrial dysfunction, reducing oxidative damage, promoting glutamate clearance and inhibiting mitochondrial-nuclear translocation of apoptosis-inducing factor, in rats with middle cerebral artery occlusion (MCAO) injury (20-25). It has also been reported that ginsenoside can promote the differentiation and proliferation of NSCs (26). However, whether its therapeutic effect on cerebral ischemia and hypoxia injury is associated with the HIF- $1 \alpha$-VEGF pathway remains to be elucidated.

The aim of the present study was to establish an OGD/R model of NSCs to investigate the synergistic effects of oxygen and glucose withdrawal, and the duration of OGD/R (2, 4 and $6 \mathrm{~h}$ ) on the proliferation, differentiation and outgrowth of NSCs. This was assessed by determining the protein levels of VEGF and HIF-1 $\alpha$, and the growth status of the NSCs to confirm the effect of ginsenoside in the promotion of NSC proliferation and differentiation by regulating the HIF-1 $\alpha$-VEGF pathway.

\section{Materials and methods}

Chemicals and reagents. Fetal bovine serum (FBS), DMEM/F12, basic fibroblast growth factor, penicillin-streptomycin liquid and trypsin were obtained from Invitrogen; Thermo Fisher Scientific, Inc. (Waltham, MA, USA); polylysine, mouse anti-bromodeoxyuridine (BrdU) antibody (cat. no. ab8152), rabbit anti-nestin antibody (cat.no.ab11306), mouse anti-neuronspecific class III $\beta$-tubulin (tuj-1) antibody (cat. no. ab52623), mouse anti-vimentin antibody (cat. no. ab8978), goat anti-rabbit IgG antibody (cat. no. ab6939), and goat anti-rat IgG antibody (cat. no. ab6717) were obtained from Abcam (Cambridge, MA, USA); 2-(4-amidinophenyl)-6-indolecarbamidine (DAPI) staining solution and HEPES were obtained from Solarbio (Beijing, China); dimethylsulfoxide (DMSO) was obtained from Amresco LLC (Solon, OH, USA); and $\beta$-glycerin sodium and 3-(4,5-dimethylthiazol-2-yl)-2,5-diphenyltetrazolium bromide (MTT) were obtained from Beyotime (Beijing, China).

Animals. A total of 4 pregnant female Sprague-Dawley rats of SPF grade were purchased from Sibeifu Experimental Animal Science and Technology Co., Ltd. (Beijing, China; no. SCXK2011-0004) on embryonic day 17 (E17). All animals were housed individually at $22 \pm 2^{\circ} \mathrm{C}$ and a relative humidity of $50 \pm 10 \%$ with a $12 \mathrm{~h} \mathrm{light} / 12 \mathrm{~h}$ dark cycle. Food and water were given ad libitum throughout the experiment. All procedures in the present study were performed in accordance with the institutional guidelines and ethics of Beijing University of Chinese Medicine (Beijing, China). All surgical procedures were performed under anesthesia and all efforts were made to minimize suffering.

Isolation and culture of NSCs. The E17 fetuses were removed from the female rats in advanced pregnancy individually following anesthetization, following which the fetuses were immediately decapitated, and the brain and its surrounding meninges were immediately removed. The primary cell cultures were established from the hippocampal tissues of the fetuses. The dissociated embryonic tissue was digested with $0.05 \%$ trypsin for $25 \mathrm{~min}$ at $37^{\circ} \mathrm{C}$, and the suspension of NSCs was passed through a 200-mesh sieve following washing with DMEM-F12 and NSC complete culture solution. Following cell counting with a light microscope (magnifcation, $\mathrm{x} 40$ ), the harvested NSCs were grown in an incubator (Thermo Fisher Scientific, Inc.) with $5 \% \mathrm{CO}_{2}$ and at a constant temperature of $37^{\circ} \mathrm{C}$. Following culture for 5-7 days, spheres of NSCs were formed in the suspension, which were mechanically dissociated into individual cells. The suspension of NSCs was then digested with $0.05 \%$ trypsin for $2 \mathrm{~min}$ at $37^{\circ} \mathrm{C}$, and cultured to a clonal density of $1 \times 10^{5}$ cells $/ \mathrm{ml}$ in $5 \% \mathrm{CO}_{2}$ at $37^{\circ} \mathrm{C}$.

MTT method. The MTT method was used to identify the optimal concentration of ginsenoside and duration of $\mathrm{OGD} / \mathrm{T}$ on the proliferation and differentiation of the NSCs. Following isolation, the single-cell suspension $\left(1 \times 10^{5}\right.$ cells $\left./ \mathrm{ml}\right)$ was cultured in a 96-well plate coated with 
Table I. Treatment groups and durations of OGD/R.

\begin{tabular}{|c|c|c|c|c|}
\hline $\begin{array}{l}\text { Fluorescent- } \\
\text { labeled } \\
\text { antibodies }\end{array}$ & Group & $\begin{array}{c}\text { Time } \\
\text { of OGD } \\
(\mathrm{h})\end{array}$ & $\begin{array}{l}\text { Time of } \\
\text { simulated } \\
\text { reperfusion } \\
\text { (h) }\end{array}$ & $\begin{array}{l}\text { Total } \\
\text { OGD/R } \\
\text { time (h) }\end{array}$ \\
\hline \multirow[t]{9}{*}{ Nestin/BrdU } & Control & 0 & 6 & 6 \\
\hline & & 0 & 8 & 8 \\
\hline & & 0 & 10 & 10 \\
\hline & Vehicle & 4 & 2 & 6 \\
\hline & & 4 & 4 & 8 \\
\hline & & 4 & 6 & 10 \\
\hline & $\begin{array}{l}\text { Ginsenoside- } \\
\text { treated }\end{array}$ & 4 & 2 & 6 \\
\hline & & 4 & 4 & 8 \\
\hline & & 4 & 6 & 10 \\
\hline \multirow[t]{9}{*}{ Nestin/tuj-1 } & Control & 0 & 6 & 6 \\
\hline & & 0 & 8 & 8 \\
\hline & & 0 & 10 & 10 \\
\hline & Vehicle & 4 & 2 & 6 \\
\hline & & 4 & 4 & 8 \\
\hline & & 4 & 6 & 10 \\
\hline & $\begin{array}{l}\text { Ginsenoside- } \\
\text { treated }\end{array}$ & 4 & 2 & 6 \\
\hline & & 4 & 4 & 8 \\
\hline & & 4 & 6 & 10 \\
\hline \multirow[t]{9}{*}{ Nestin/vimentin } & Control & 0 & 6 & 6 \\
\hline & & 0 & 8 & 8 \\
\hline & & 0 & 10 & 10 \\
\hline & Vehicle & 4 & 2 & 6 \\
\hline & & 4 & 4 & 8 \\
\hline & & 4 & 6 & 10 \\
\hline & $\begin{array}{l}\text { Ginsenoside- } \\
\text { treated }\end{array}$ & 4 & 2 & 6 \\
\hline & & 4 & 4 & 8 \\
\hline & & 4 & 6 & 10 \\
\hline
\end{tabular}

OGD/R, oxygen-glucose deprivation/reperfusion; tuj-1, neuron-specific class III $\beta$-tubulin; BrdU, bromodeoxyuridine

poly-lysine $(0.01 \%$, wt/vol). Following culture in a humidified $5 \% \mathrm{CO}_{2} / 95 \%$ air incubator at $37^{\circ} \mathrm{C}$ for $24 \mathrm{~h}$, ginsenoside at 10 concentrations $(0.25,0.5,1,2.5,6.25,12.5,25,50$, 100 and $200 \mu \mathrm{g} / \mathrm{ml}$ ) were respectively added into the plate wells for 3 days. In the control group, ginsenoside was added into complete medium only. The optical density (OD) values of each well were detected on a microplate reader at a wavelength of $570 \mathrm{~nm}$, and the optimal concentration was calculated according to the OD values.

The single-cell suspension $\left(5 \times 10^{5}\right.$ cells $\left./ \mathrm{ml}\right)$ was cultured in 96 -well plates coated with poly-lysine $(0.01 \%$, wt/vol) with the optimal dose of ginsenoside. The cells were cultured for eight periods of time $(1,3,6,12,24,48,72$ and $96 \mathrm{~h})$ in the control group and ginsenoside group. The OD values of each well were detected on a microplate spectrophotometer, and the optimal time-period was calculated according to the OD values.
OGD/R model establishment and ginsenoside administration. Three groups, namely the control group, vehicle group and ginsenoside-treated group, were included in the present study. The OGD/R model was established as reported previously $(27,28)$ with minor modification. The cells in the control group were incubated with glucose Earle's balanced salt solution (BSS), and the cells in the vehicle group and ginsenoside-treated group were incubated with glucose-free Earle's BSS. The cells were then immediately transferred to a humidified anaerobic chamber for $4 \mathrm{~h}$ with $94 \% \mathrm{~N}_{2}, 5 \% \mathrm{CO}_{2}$ and $1 \% \mathrm{O}_{2}$ at $37^{\circ} \mathrm{C}$. During the OGD process, ginsenoside $(1 \mu \mathrm{g} / \mathrm{ml})$ was added to the Earle's BSS in the ginsenosidetreated group. For terminating cell OGD and perfusion, the cells were then cultured in neurobasal medium and the supplements under a humidified atmosphere of $5 \% \mathrm{CO}_{2}$ and $95 \%$ air at $37^{\circ} \mathrm{C}$, during which ginsenoside $(1 \mu \mathrm{g} / \mathrm{ml})$ was added to the medium in the ginsenoside-treated group again. The cells were then used for the subsequent experiments of western blot analysis, enzyme-linked immunosorbent assay (ELISA) and double-labeling immunofluorescence.

Western blot analysis. Western blot analysis was performed on the extracts prepared from the different groups of NSCs, and the cell lysates collected and analyzed for evaluating the expression of HIF-1 $\alpha$. PMSF ( $2 \mu \mathrm{l} ; 0.25 \mathrm{~mol} / \mathrm{l})$, cytoplasmic extraction reagent (CER)I, CERII and nuclear extraction reagent were added in sequence to the drying cell lysates, and the nucleoprotein was collected from the supernatant following repeated vortexing and centrifugation $(16,000 \mathrm{x} \mathrm{g}$, $\left.4^{\circ} \mathrm{C}, 10 \mathrm{~min}\right)$.

The concentrations of the nucleoprotein were determined with BCA protein assay kit. The nucleoprotein $(30 \mu \mathrm{g})$ was then resolved by sodium dodecyl sulfate-PAGE, and then transferred onto a PVDF membrane (EMD Millipore, Billerica, MA, USA). The membrane was incubated with primary antibodies HIF-1 $\alpha$ (1:500, cat. no. ab113642; Abcam, Beijing, China) in saline/Tween-20 buffered by $1 \%$ bovine serum albumin (Thermo Fisher Scientific, Inc.)/phosphate at $4^{\circ} \mathrm{C}$ overnight, following the incubation with secondary antibodies goat anti-mouse IgG (1:30, cat. no. SA00001-1; ProteinTech, Hubei, China) at room temperature for $2 \mathrm{~h}$. The protein signals were detected using the enhanced chemiluminescence method.

ELISA. The cell-free supernatants of the treated and untreated NSC suspensions in each group were collected $72 \mathrm{~h}$ following treatment with ginsenoside, and were used to measure the production of VEGF using an ELISA kit according to the manufacturer's protocol. The levels of VEGF were determined and calculated according to the results of the OD value at $450 \mathrm{~nm}$.

Immunofluorescence staining. The design of the fluorescent labeling experiment for each group is shown in Table I. The cells $\left(1 \times 10^{5} / \mathrm{ml}\right)$ were plated onto coverslips for $24 \mathrm{~h}$, and the coverslips were respectively coated with poly-D-lysine and immunofluorescent labeling for nestin/BrdU, nestin/vimentin, and nestin/tuj-1, in order to detect the self-renewal and proliferation, astrocytic differentiation, and neuronal differentiation of the NSCs. The primary antibodies used comprised nestin 
and BrdU antibodies (1:300 and 1:400, respectively), nestin and vimentin antibodies (1:400 and 1:500, respectively), and nestin and tuj-1 antibodies (1:400 each). Goat anti-rabbit IgG-Cy3 (1:50) and goat anti-mouse IgG-FITC (1:50) were used as the secondary antibodies. Counterstaining of cell nuclei was then performed with DAPI $(100 \mathrm{ng} / \mathrm{ml})$ for $10 \mathrm{~min}$. Viable cells were then counted with laser-scanning confocal microscopy (Olympus, Tokyo, Japan).

Image analysis and statistical analysis. Image-Pro Plus software (version 6.0; Media Cybernetics, Inc., Rockville, MD, USA) was used to analyze the number, density and OD of the positive cells in the fluorescence images. All data were processed with the use of SPSS 20.0 (IBM SPSS, Armonk, NY, USA). The data are expressed as the mean \pm standard deviation. The significance of variables was determined using a paired-sample t-test. $\mathrm{P}<0.05$ was considered to indicate a statistically significant difference.

\section{Results}

Identification of NSCs. Initially, the cells in the suspension prepared during the dissection of the fetuses were single, small and transparent, and were round or elliptical in shape with little protuberance and a glossy appearance. Cell clone spheres began to form at $24 \mathrm{~h}$ post-dissection. They became round in shape following 3-4 days and were passaged at 7-8 days (Fig. 1). The primary neurospheres had a regular morphology without irregularly sized processes, and their volumes increased with culture duration, the same as was observed in the passaged cell clone spheres.

The expression of nestin was measured following 4 days of cell culture (Fig. 1C) The nestin-positive cells varied in volume, with or without apophyses, and positive red staining was observed in the cytoplasm. The stained cells were round or oval in shape, and their nuclear zones were unstained and often shifted to one side of the cell.

Positive dark green staining was observed in the nuclear region, which confirmed the expression of BrdU, whereas less staining was observed in the cytoplasm (Fig. 1D) The BrdU-positive cells were found to be small in volume with apophyses.

A green color was observed in the cytoplasm, which confirmed the expression of tuj-1 (Fig. 1E). The nuclear region of tuj-1-positive cells was unstained and often shifted to one side of the cell. The tuj-1-positive cells were small in volume, round in shape and featured apophyses.

The expression of vimentin, confirmed by green staining, was observed in the cytoplasm (Fig. 1F). The nuclear zones of the vimentin-positive cells were unstained and often shifted to one side of the cell.

The above-mentioned results indicated that the NSCs were identified by the positive expression of nestin, NSC proliferation was assessed according to the positive expression of BrdU, and NSC differentiation was identified by the enhanced expression of tuj-1 and vimentin.

Determination of the optimal dose and $O G D / R$ duration for the effect of ginsenoside on NSC proliferation and differentiation. The effects of ginsenoside on the proliferation and
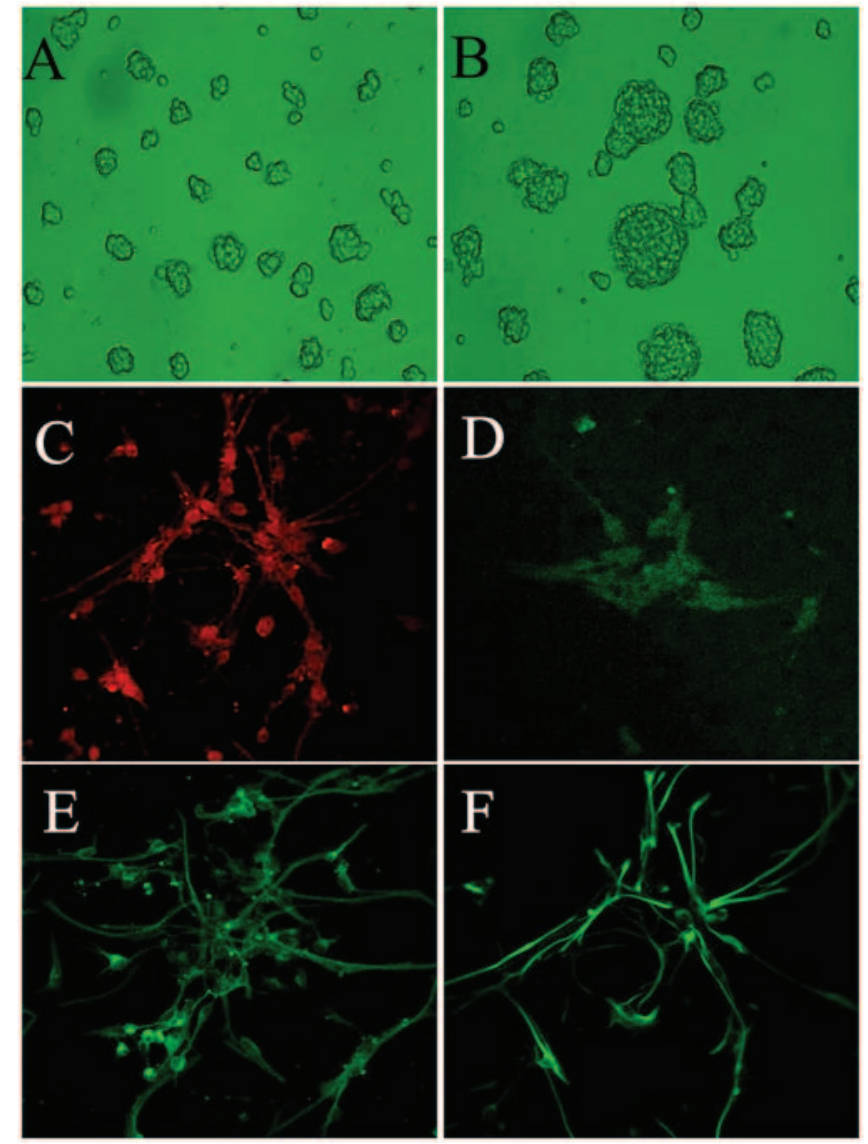

Figure 1. Identification of NSCs and its differentiated cells. (A) Primary culture of cell clone spheres. (B) Passage culture of cell clone spheres. Immunofluorescence images of the NSCs to show (C) nestin, (D) BrdU, (E) tuj-1 and (F) vimentin (magnification, x200). Primary neurospheres had a regular morphology without irregularly sized processes. Volume increased with the culture time, as with the passaged cell clone spheres. The immunofluorescence staining showed that the cells were nestin-positive, with red fluorescent in the cytoplasm. The cells shown to be BrdU-positive following 4 days with proliferation condition medium, exhibited green fluorescence in the cytoplasm. NSCs, neural stem cells; BrdU; bromodeoxyuridine; tuj1, neuron-specific class III $\beta$-tubulin.

differentiation of NSCs, which were cultured with different concentrations of ginsenoside for 3 days, are shown in Fig. 2A. It was found that, compared with the control group, the OD values of the NSCs were significantly increased by ginsenoside at concentrations of $1,2.5$ and $6.25 \mu \mathrm{g} / \mathrm{ml}(\mathrm{P}<0.01)$, according to the MTT assay. In addition, the OD value of the NSCs reached its highest level at a concentration of $1 \mu \mathrm{g} / \mathrm{ml}$, which suggested that this may be the optimal dose for affecting the proliferation and differentiation of the NSCs.

The effect of ginsenoside at a dose of $1 \mu \mathrm{g} / \mathrm{ml}$ with different OGD/R durations on the proliferation and differentiation of NSCs is shown in Fig. 2B. Compared with that in the control group, the OD value of the NSCs was significantly higher between 3 and $72 \mathrm{~h}$ in the ginsenoside-treated group, which indicated that ginsenoside promoted the proliferation and differentiation of NSCs in this period of time; however, it was found that this effect was optimal within $24 \mathrm{~h}$, following which it weakened with time.

Effect of ginsenoside on the protein expression of HIF-1. The expression of HIF-1 $\alpha$ in the ginsenoside-treated group was 

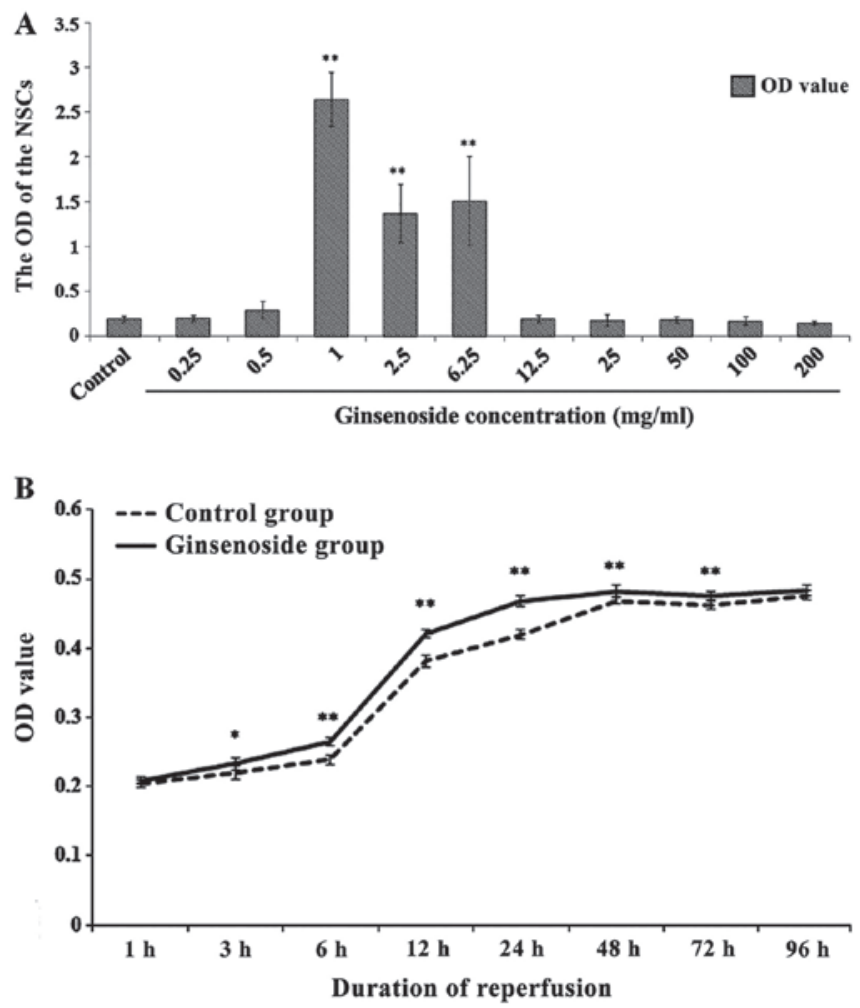

Figure 2. Effects of ginsenoside on proliferation and differentiation NSCs. Effects of different (A) concentrations of ginsenoside and (B) durations of reperfusion. The values were analyzed using a t-test and expressed as the mean \pm standard deviation $(\mathrm{n}=8)$. ${ }^{*} \mathrm{P}<0.05$ and ${ }^{* *} \mathrm{P}<0.01$ vs. control groups. NSCs, neural stem cells; OD, optical density.

confirmed using western blot analysis (Fig. 3). The results of the western blot analysis showed that, compared with the vehicle group, the expression of HIF-1 $\alpha$ in the OGD/R-treated NSCs in the ginsenoside-treated group was significantly increased $(\mathrm{P}<0.05)$. The difference between the expression levels of HIF-1 $\alpha$ in the NSCs of the vehicle and ginsenoside-treated groups gradually increased with the duration of simulated reperfusion, and were highest at $6 \mathrm{~h}$.

Effect of ginsenoside on the protein expression of VEGF. The results of the ELISA showed that the protein expression of VEGF was at a low level in the NSCs of the control group (Fig. 4), however, the level was markedly upregulated when the cells were damaged by hypoxia $(\mathrm{P}<0.05)$. The protein levels of VEGF in the NSCs of the ginsenoside-treated group were significantly increased with the duration of simulated reperfusion $(\mathrm{P}<0.05)$, showing the same results as with HIF-1 $\alpha$.

Effect of ginsenoside on the proliferation and differentiation of NSCs. The results of the immunofluorescence staining experiments of the effects of ginsenoside on the proliferation and differentiation of NSCs are shown in Figs. 5-7, which include a series of findings. Firstly, it was found that the expression of nestin-positive cells was in the cytoplasm, and the body of the cell was round or oval in shape with no or few neurites; the number of the nestin-positive cells in the vehicle group was lower, compared with that in the control group, and the cells had no or few neurites. However, it was found that, in the ginsenoside-treated group, the number of nestin-
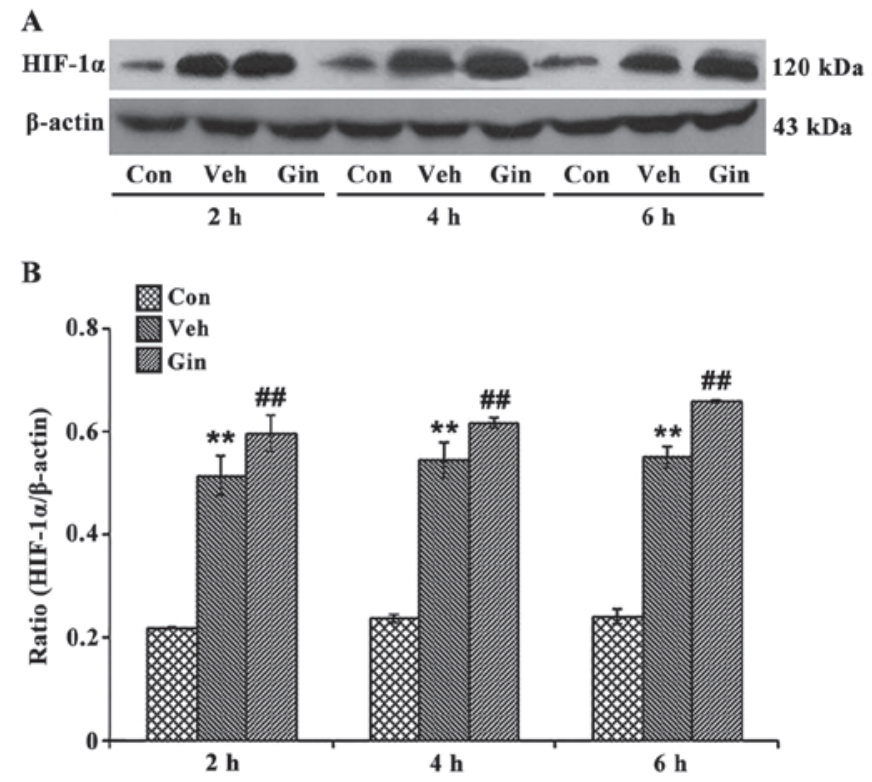

Figure 3. Expression of HIF-1 $\alpha$ in the neural stem cells. (A) Representative image of the protein expression of HIF-1 $\alpha$. (B) Bar graph quantifying the protein levels of HIF-1 $\alpha$. The results are expressed as the mean \pm standard deviation. $\beta$-actin was used as the internal control. There were three time points of reperfusion (2, 4 and $6 \mathrm{~h}$ ) for the groups: $4 \mathrm{~h}$ of OGD and simulated reperfusion for $2 \mathrm{~h} ; 4 \mathrm{~h}$ of OGD and simulated reperfusion for $4 \mathrm{~h} ; 4 \mathrm{~h}$ of OGD and simulated reperfusion for $6 \mathrm{~h}$. ${ }^{* *} \mathrm{P}<0.01$ vs. Con; ${ }^{* \#} \mathrm{P}<0.01$ vs. Veh. Con, control group; Veh, vehicle group; Gin, ginsenoside-treated group; HIF-1 $\alpha$, of hypoxia-inducible factor- $1 \alpha$.

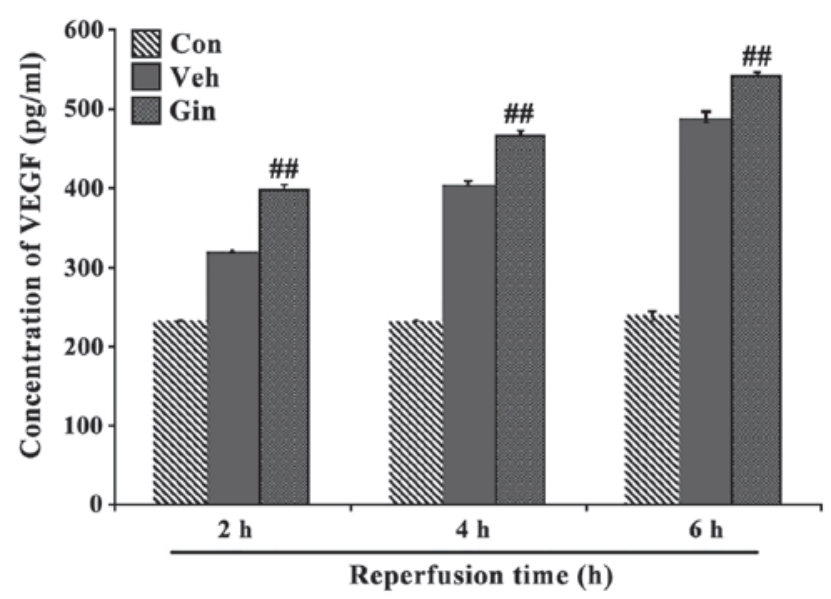

Figure 4. Concentrations of VEGF. Concentrations of VEGF (pg/ml) were determined in the three groups. ${ }^{\# \#} \mathrm{P}<0.05$ vs. Veh. VEGF, vascular endothelial growth factor; Con, control group; Veh, vehicle group; Gin, ginsenoside-treated group.

positive cells increased and the majority of the cells contained neurites. In the ginsenoside-treated group, the BrdU staining was present mainly in the nuclei of the nestin-positive cells with minimal staining in the cytoplasm. The cells were also smaller in volume and contained neurites. Secondly, it was found that the number of BrdU-positive cells decreased and the cells contained no or few protrusions in the vehicle group, compared with those in the control group. The number of BrdU-positive cells was increased and the cells contained increased neurites. The tuj-1 staining was observed mainly in the cytoplasm and the cells were smaller in volume with 


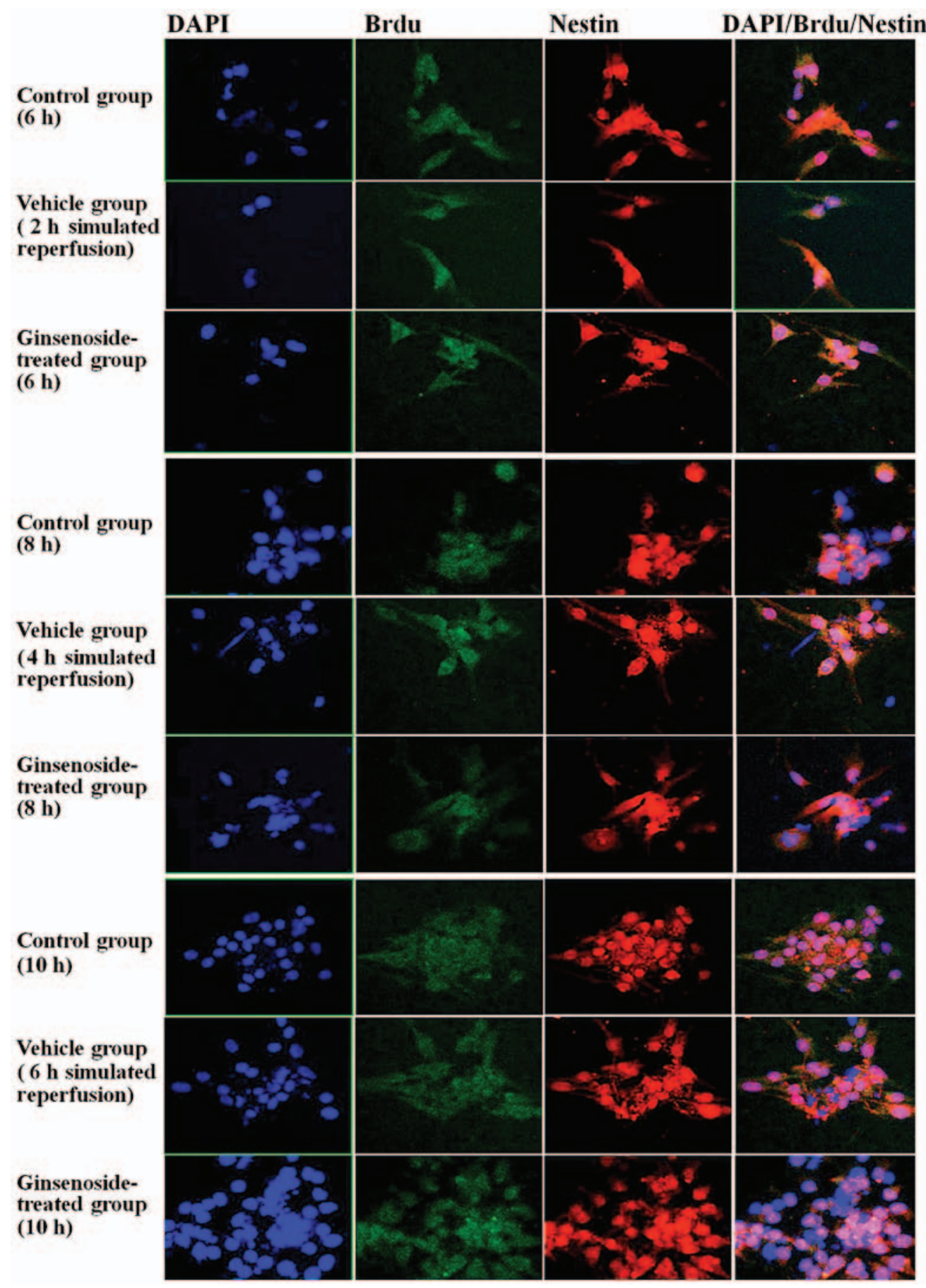

Figure 5. Morphological observations of the number, optical density and area density of nestin/BrdU double-labeled positive cells following 2,4 and $6 \mathrm{~h}$ of reperfusion. From left to right (x200): DAPI (blue), BrdU (green), Nestin (red), Merge. DAPI, 2-(4-amidinophenyl)-6-indolecarbamidine; BrdU, bromodeoxyuridine.

neurites. In the vehicle group, the number of tuj-1-positive cells was reduced and the volumes of the cells were smaller, compared with those in the ginsenoside-treated group, in which the volume of cells and the number of neurites were increased, with aggregation of vimentin staining in the cytoplasm. Thirdly, it was found that, in the vehicle group, the number of vimentin-positive cells was decreased and the cells were smaller in volume with fewer neurites, compared with those in the control group. However, in the ginsenosidetreated group, the number of vimentin-positive cells and the volume of the cells were increased and the cells contained more neurites.
The statistical results showed that, compared with those in the control group, the number of positive cells, OD and area density were significantly decreased between 2 and $4 \mathrm{~h}$ of reoxygenation $(\mathrm{P}<0.01)$ in the vehicle group, whereas the positive cell number, OD and area density were significantly increased $(\mathrm{P}<0.01)$ in the ginsenoside-treated group, compared with those in the vehicle group. The statistical results also showed that the number of positive cells, OD and area density were decreased at $6 \mathrm{~h}$ of reoxygenation $(\mathrm{P}>0.05)$ in the vehicle group, whereas the number of positive cells, OD and density were increased significantly $(\mathrm{P}<0.01)$, compared with those in the vehicle groups (Figs. 8-10). 


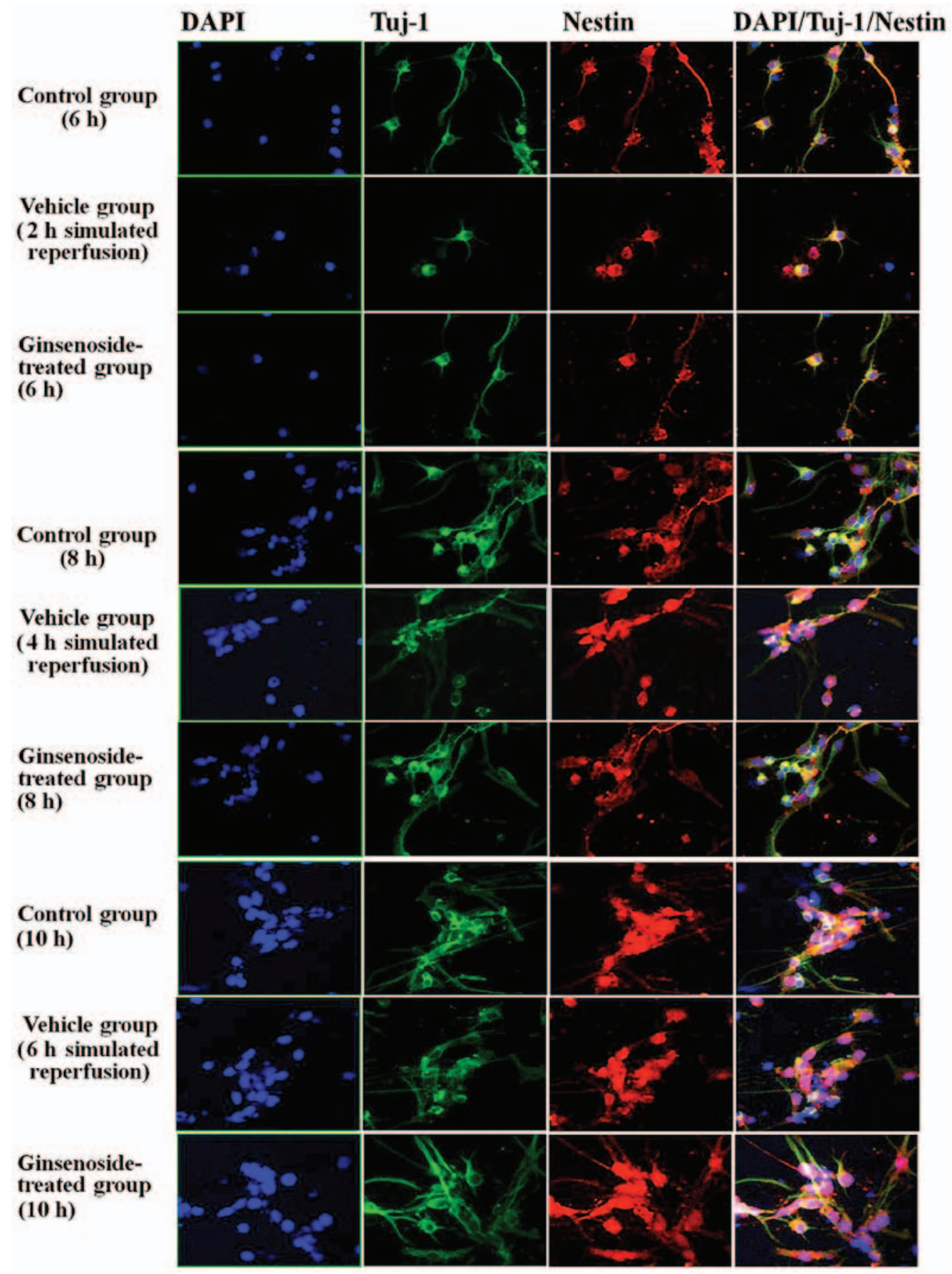

Figure 6. Morphological observation of the number, optical density and density of the nestin/tuj-1 double-labeled positive cells for 2,4 and $6 \mathrm{~h}$ of reperfusion. From left to right (x200): DAPI (blue), tuj-1 (green), nestin (red), merge. DAPI, 2-(4-amidinophenyl)-6-indolecarbamidine; BrdU, bromodeoxyuridine; tuj-1, neuron-specific class III $\beta$-tubulin.

\section{Discussion}

Characterized by a loss of neurons and glial cells in the brain, stroke, or traumatic brain injury, can lead to the death of brain cells. NSCs can proliferate and migrate into the injured region of the brain, and differentiate into the corresponding nerve cells to become involved in the formation of neural circuits, and the promotion of structural and functional repair of the injured brain $(29,30)$. NSCs may also be involved in treatment of the brain degeneration associated with certain diseases, including Parkinson's disease and Alzheimer's disease (31-33). Ginsenoside can exert effects promoting the proliferation and differentiation of NSCs (34), however, the molecular mechanism of this effect remains to be elucidated.

Acting upstream of the Wnt/ $\beta$-catenin pathway, HIF- $1 \alpha$ may contribute to the production of VEGF (35), which is an important signaling molecule in angiogenesis and neurogenesis (11). As a master regulator of cellular adaptation to hypoxia, HIF-1 $\alpha$ is considered as a potent therapeutic target in cerebral ischemia. HIF-1 $\alpha$ can be activated by the reduction in cellular oxygen supply caused by MCAO, followed by the secretion of its downstream protein, VEGF; this is a critical factor in angiogenesis, and can be produced and secreted by various types of cell to increase capillary permeability and stimulate 


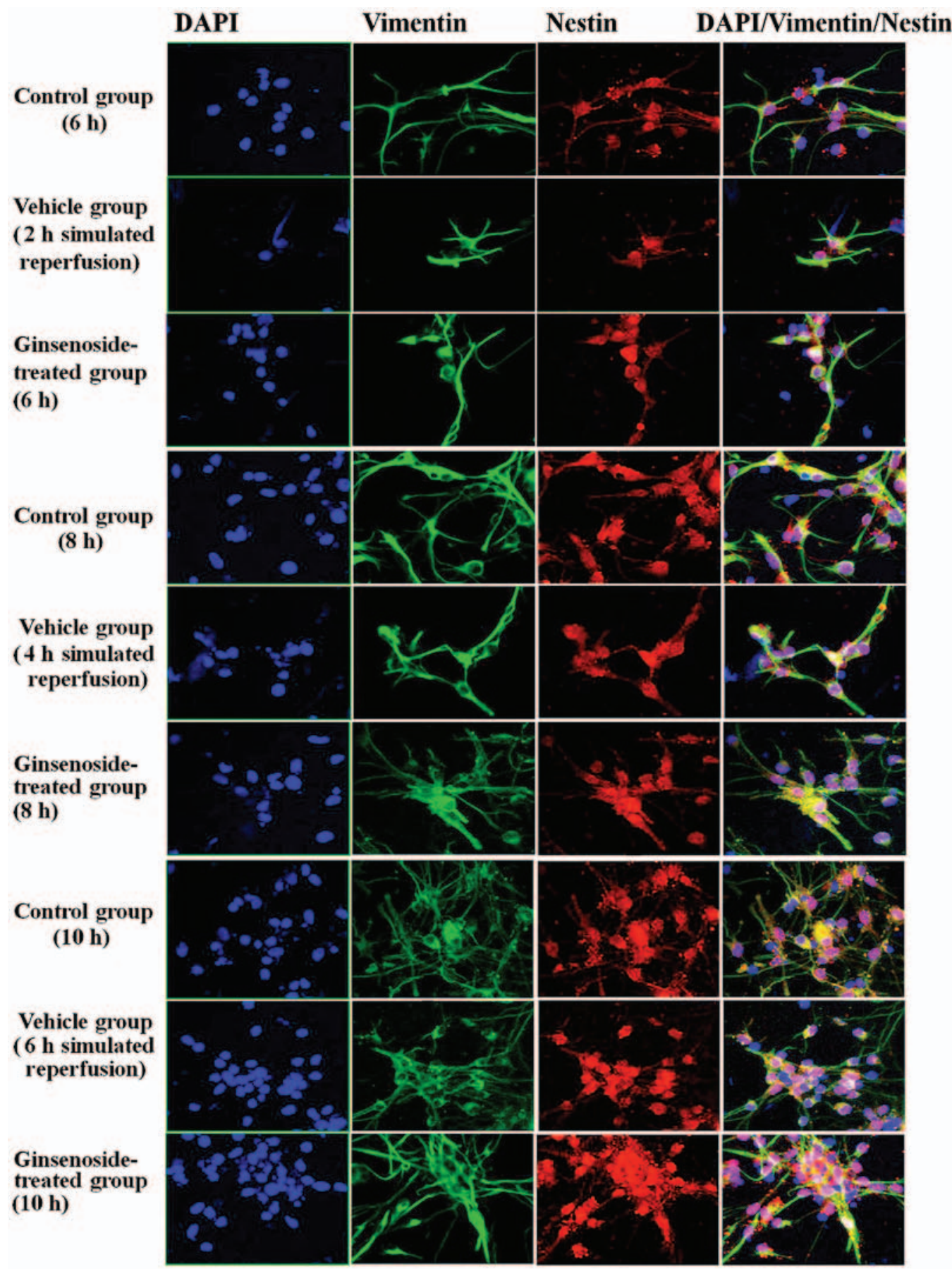

Figure 7. Morphological observation of the number, optical density and density of the nestin/vimentin double-labeled positive cells for 2,4 and $6 \mathrm{~h}$ of reperfusion. From left to right (x200): DAPI (blue), vimentin (green), nestin (red), Merge. DAPI, 2-(4-amidinophenyl)-6-indolecarbamidine; BrdU, bromodeoxyuridine.

the proliferation of endothelial cells (36-38). A previous study using a neonatal hypoxia/ischemia model also confirmed the effects of ginsenoside on inhibiting apoptosis, and increasing the expression of HIF-1 $\alpha$ and VEGF in injured brain tissue. Tang et al concluded that $\operatorname{Rg} 1$ has a neuroprotective role in brain repair following neonatal hypoxia/ischemia, and that HIF- $1 \alpha$ is a potential target for therapeutic intervention in neonates with hypoxic/ischemic brain injury (39). As a type of intermediate filament protein, nestin is a specific marker for embryonic NSCs. Previous in vitro studies have shown that NSCs are characterized by the expression of nestin, capacity for continuous proliferation, self-renewal, and multidirectional differentiation under specific conditions (16,40-42). The results of the present study showed that ginsenoside at certain concentrations increased the number of nestin-expressing NSCs, which suggested that ginsenoside may promote the survival, proliferation and self-renewal of NSCs by inducing the production of undifferentiated and nestin-positive cells. The results from the experiments on ginsenoside-treated BrdU-positive NSCs suggested that ginsenoside accelerated the proliferation of NSCs. The results of the experiments on rats in the present study also suggested that ginsenoside promoted the differentiation of embryonic cortical NSCs into tuj-1-positive neurons and vimentin-positive astrocytes. 

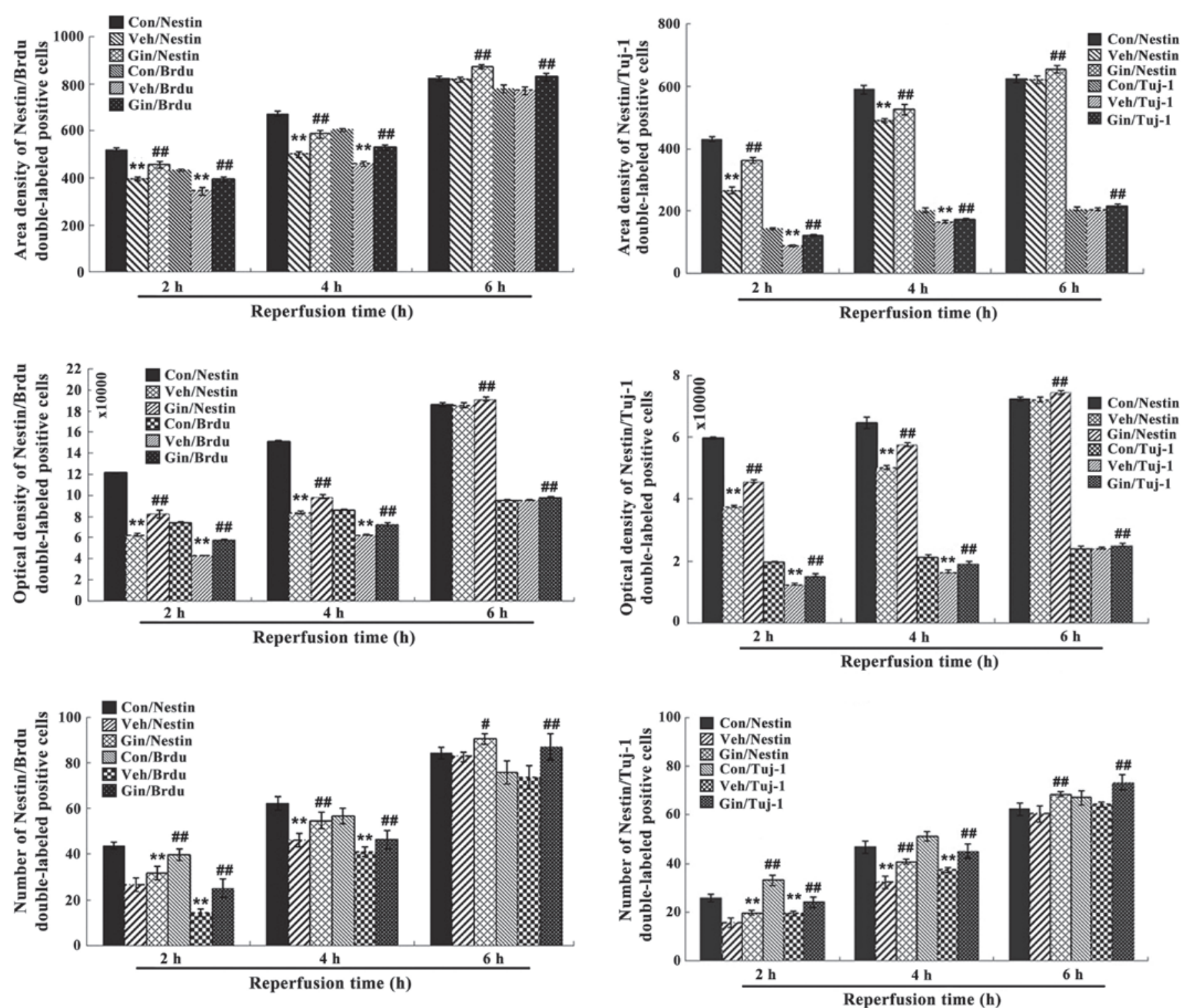

Figure 8. Nestin/BrdU double-labeled immunochemical staining results following 2, 4 and $6 \mathrm{~h}$ of reperfusion. Results for area density, optical density and positive number are shown. ${ }^{* *} \mathrm{P}<0.01$ vs. Con; ${ }^{\#} \mathrm{P}<0.05$ and ${ }^{\# \#} \mathrm{P}<0.01$ vs. Veh. Compared with those in the control group, the number of positive cells, optical density and area density were decreased significantly between 2 and $4 \mathrm{~h}$ of reoxygenation $(\mathrm{P}<0.01)$ in the vehicle group and were significantly increased $(\mathrm{P}<0.01)$ in the ginsenoside-treated group, compared with those in the vehicle group. The statistical results showed that the number of positive cells, optical density and area density were decreased following $6 \mathrm{~h}$ of reoxygenation $(\mathrm{P}>0.05)$ in the vehicle group, but were significantly increased $(\mathrm{P}<0.01)$ in the ginsenoside-treated group, compared with those in the vehicle groups. Veh. Con, control group; Veh, vehicle group; Gin, ginsenoside-treated group; BrdU, bromodeoxyuridine.

The increased number of positive NSCs, higher OD values and area density in the ginsenoside-treated group in the present study suggested that ginsenoside may protect the nerve cells from injury induced by OGD/R. The increase in the number of positive NSCs, in addition to the increased duration for reoxygenation may be due to the hypoxia-induced activation of HIF- $1 \alpha$ and the formation of HIF-1, which is the result of the migration of activated HIF- $1 \alpha$ to the nucleus and combing with HIF-1 $\beta$. HIF-1 may regulate the production of VEGF protein following transcription and translation. The stability of VEGF protein can increase under anoxic conditions, and

Figure 9. Nestin/tuj-1 double-labeled immunochemical staining results of the control group, vehicle group and ginsenoside-treated group following 2 , 4 and 6 h. of reperfusion. ${ }^{* *} \mathrm{P}<0.01$ vs. Con; ${ }^{\# \#} \mathrm{P}<0.01$ vs. Veh. Compared with those in the control group, the number of positive cells, optical density and area density were significantly decreased between 2 and $4 \mathrm{~h}$ of reoxygenation $(\mathrm{P}<0.01)$ in the vehicle group, but were increased significantly $(\mathrm{P}<0.01)$ in the ginsenoside-treated group, compared with those in the vehicle group. The number of positive cells, optical density and area density were decreased following $6 \mathrm{~h}$ of reoxygenation $(\mathrm{P}>0.05)$ in the vehicle group, but were increased significantly $(\mathrm{P}<0.01)$ in the ginsenoside-treated group, compared with those in the vehicle group. Veh. Con, control group; Veh, vehicle group; Gin, ginsenoside-treated group; BrdU, bromodeoxyuridine; tuj-1, neuronspecific class III $\beta$-tubulin.

the combination of its receptors and specific ligands can promote angiogenesis, proliferation and migration of nerve cells, neurotrophic activity and nerve regeneration. VEGF can also promote NSC survival in the brain and stimulate neurogenesis in vitro and in vivo (15-17). The change in the number of positive NSCs in the ginsenoside-treated group in the present study showed that ginsenoside may increase the differentiation and proliferation of NSCs. The increase in the expression levels of VEGF and HIF-1 $\alpha$ by ginsenoside indicated that ginsenoside may exert cerebral protection by 

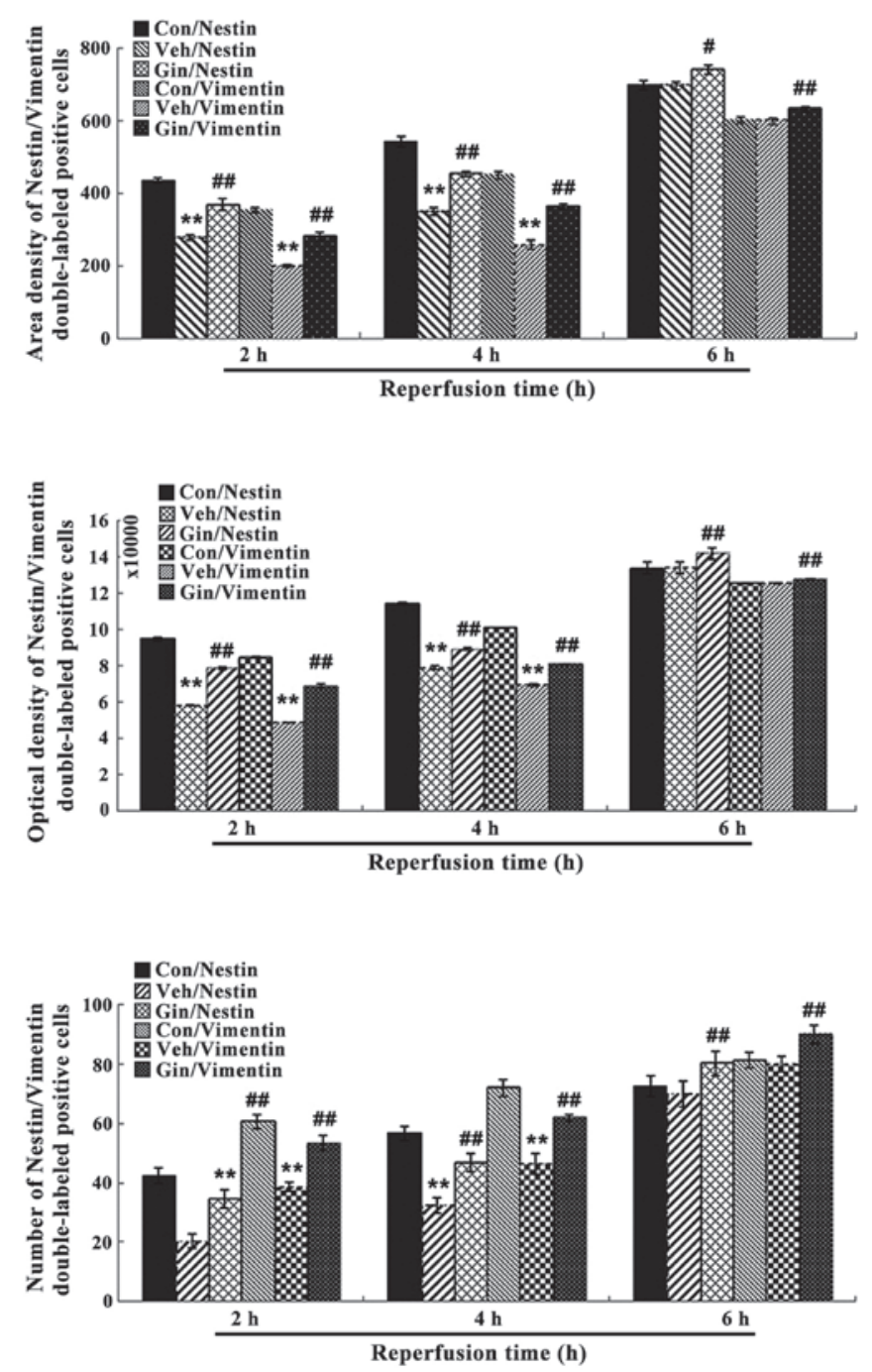

Figure 10. Nestin/vimentin double-labeled immunochemical staining results of the control group, vehicle group, and ginsenoside-treated group following 2,4 and $6 \mathrm{~h}$ of reperfusion. ${ }^{* *} \mathrm{P}<0.01$ vs. Con; ${ }^{\#} \mathrm{P}<0.05 ;{ }^{\# \#} \mathrm{P}<0.01$ vs. Veh. Compared with those in the control group, the number of positive cells, optical density and area density were decreased significantly between 2 and 4 h of reoxygenation $(\mathrm{P}<0.01)$ in the vehicle group, but were increased significantly $(\mathrm{P}<0.01)$ in the ginsenoside-treated group, compared with those in the vehicle group. The number of positive cells, optical density and area density were decreased following $6 \mathrm{~h}$ of reoxygenation $(\mathrm{P}>0.05)$ in the vehicle group, but were increased significantly $(\mathrm{P}<0.01)$ in the ginsenoside-treated group, compared with those in the vehicle group. Veh. Con, control group; Veh, vehicle group; Gin, ginsenoside-treated group; BrdU, bromodeoxyuridine.

promoting the protein expression of HIF-1 $\alpha$, followed by initiating the downstream VEGF pathway at the early phase of OGD/R.

In conclusion, the results from the experiments in the present study suggested that ginsenoside may maintain the replication of NSCs, promote NSC proliferation, promote their differentiation into neurons and astrocytes. This indicated that ginsenoside exerted a protective effect on the NSCs injured by ischemia-reperfusion through the HIF- $1 \alpha$-VEGF pathway. The results of the present study provide a theoretical basis for the possible treatment of ischemic stroke with ginsenoside, and provide scope for investigating the mechanisms of traditional Chinese medicine preparations in treating diseases associated with the nervous system in novel drugs.

\section{Acknowledgements}

This study was funded by the National Natural Science Foundation of China (grant no. 81373830).

\section{References}

1. Shi Q, Zhang P, Zhang J, Chen X, Lu H, Tian Y, Parker TL and Liu Y: Adenovirus-mediated brain-derived neurotrophic factor expression regulated by hypoxia response element protects brain from injury of transient middle cerebral artery occlusion in mice. Neurosci Lett 465: 220-225, 2009.

2. Mehta SL, Manhas N and Raghubir R: Molecular targets in cerebral ischemia for developing novel therapeutics. Brain Res Brain Res Rev 54: 34-66, 2007.

3. Peruzzotti-JamettiL,DonegáM,GiustoE,MallucciG,MarchettiB and Pluchino S: The role of the immune system in central nervous system plasticity after acute injury. Neuroscience 283: 210-221, 2014.

4. Kalluri HS, Eickstaedt J and Dempsey RJ: Oxygen glucose deprivation inhibits the growth and ERK phosphorylation of neural progenitor cells in vitro. Neurosci Lett 426: 145-148, 2007.

5. Lipton P: Ischemic cell death in brain neurons. Physiol Rev 79: 1431-1568, 1999.

6. de Pablo Y, Nilsson M, Pekna M and Pekny M: Intermediate filaments are important for astrocyte response to oxidative stress induced by oxygen-glucose deprivation and reperfusion. Histochem Cell Biol 140: 81-91, 2013.

7. Reynolds BA and Weiss S: Generation of neurons and astrocytes from isolated cells of the adult mammalian central nervous system. Science 255: 1707-1710, 1992.

8. Alvarez-Buylla A and Lim DA: For the long run: Maintaining germinal niches in the adult brain. Neuron 41: 683-686, 2004.

9. Bacigaluppi M, Pluchino S, Peruzzotti-Jametti L, Kilic E, Kilic U, Salani G, Brambilla E, West MJ, Comi G, Martino G, et al: Delayed post-ischaemic neuroprotection following systemic neural stem cell transplantation involves multiple mechanisms. Brain 132: 2239-2251, 2009.

10. Jin K, Mao X, Xie L, Galvan V, Lai B, Wang Y, Gorostiza O, Wang $\mathrm{X}$ and Greenberg DA: Transplantation of human neural precursor cells in Matrigel scaffolding improves outcome from focal cerebral ischemia after delayed postischemic treatment in rats. J Cereb Blood Flow Metab 30: 534-544, 2010.

11. Sun J, Sha B, Zhou W and Yang Y: VEGF-mediated angiogenesis stimulates neural stem cell proliferation and differentiation in the premature brain. Biochem Biophys Res Commun 394: 146-152, 2010.

12. Blancher C, Moore JW, Talks KL, Houlbrook S and Harris AL: Relationship of hypoxia-inducible factor (HIF)-1alpha and HIF-2alpha expression to vascular endothelial growth factor induction and hypoxia survival in human breast cancer cell lines. Cancer Res 60: 7106-7113, 2000.

13. Ben-Yosef Y, Lahat N, Shapiro S, Bitterman H and Miller A: Regulation of endothelial matrix metalloproteinase-2 by hypoxia/reoxygenation. Circ Res 90: 784-791, 2002.

14. Olsson AK, Dimberg A, Kreuger J and Claesson-Welsh L: VEGF receptor signalling - in control of vascular function. Nat Rev Mol Cell Biol 7: 359-371, 2006.

15. Sun Y, Jin K, Xie L, Childs J, Mao XO, Logvinova A and Greenberg DA: VEGF-induced neuroprotection, neurogenesis, and angiogenesis after focal cerebral ischemia. J Clin Invest 111: 1843-1851, 2003.

16. Jin K, Zhu Y, Sun Y, Mao XO, Xie L and Greenberg DA: Vascular endothelial growth factor (VEGF) stimulates neurogenesis in vitro and in vivo. Proc Natl Acad Sci USA 99: 11946-11950, 2002.

17. Wada T, Haigh JJ, Ema M, Hitoshi S, Chaddah R, Rossant J, Nagy A and van der Kooy D: Vascular endothelial growth factor directly inhibits primitive neural stem cell survival but promotes definitive neural stem cell survival. J Neurosci 26: 6803-6812, 2006.

18. Liu X, Wang L, Wen A, Yang J, Yan Y, Song Y, Liu X, Ren H, Wu Y, Li Z, et al: Ginsenoside-Rd improves outcome of acute ischaemic stroke - a randomized, double-blind, placebo-controlled, multicenter trial. Eur J Neurol 19: 855-863, 2012.

19. Liu X, Xia J, Wang L, Song Y, Yang J, Yan Y, Ren H and Zhao G: Efficacy and safety of ginsenoside-Rd for acute ischaemic stroke: A randomized, double-blind, placebo-controlled, phase II multicenter trial. Eur J Neurol 16: 569-575, 2009. 
20. Ye R, Yang Q, Kong X, Han J, Zhang X, Zhang Y, Li P, Liu J, Shi M, Xiong L, et al: Ginsenoside Rd attenuates early oxidative damage and sequential inflammatory response after transient focal ischemia in rats. Neurochem Int 58: 391-398, 2011.

21. Ye R, Zhang X, Kong X, Han J, Yang Q, Zhang Y, Chen Y, Li P Liu J, Shi M, et al: Ginsenoside Rd attenuates mitochondrial dysfunction and sequential apoptosis after transient focal ischemia. Neuroscience 178: 169-180, 2011.

22. Ye R, Kong X, Yang Q, Zhang Y, Han J and Zhao G: Ginsenoside Rd attenuates redox imbalance and improves stroke outcome after focal cerebral ischemia in aged mice. Neuropharmacology 61 : 815-824, 2011

23. Ye R, Kong X, Yang Q,Zhang Y,Han J, Li P, Xiong L and Zhao G: Ginsenoside Rd in experimental stroke: Superior neuroprotective efficacy with a wide therapeutic window. Neurotherapeutics 8: 515-525, 2011.

24. Hu G, Wu Z, Yang F, Zhao H, Liu X, Deng Y, Shi M and Zhao G: Ginsenoside Rd blocks AIF mitochondrio-nuclear translocation and NF- $\kappa \mathrm{B}$ nuclear accumulation by inhibiting poly(ADP-ribose) polymerase-1 after focal cerebral ischemia in rats. Neurol Sci 34 2101-2106, 2013

25. Zhang X, Shi M, Bjørås M, Wang W, Zhang G, Han J, Liu Z, Zhang Y, Wang B, Chen J, et al: Ginsenoside Rd promotes glutamate clearance by up-regulating glial glutamate transporter GLT-1 via PI3K/AKT and ERK1/2 pathways. Front Pharmacol 4: 152, 2013.

26. Si YC, Li Q, Xie CE, Niu X, Xia XH and Yu CY: Chinese herbs and their active ingredients for activating xue (blood) promote the proliferation and differentiation of neural stem cells and mesenchymal stem cells. Chin Med 9: 13, 2014.

27. Lin D, Li G and Zuo Z: Volatile anesthetic post-treatment induces protection via inhibition of glycogen synthase kinase $3 \beta$ in human neuron-like cells. Neuroscience 179: 73-79, 2011.

28. Yan W, Fang Z, Yang Q, Dong H, Lu Y, Lei C and Xiong L: SirT1 mediates hyperbaric oxygen preconditioning-induced ischemic tolerance in rat brain. J Cereb Blood Flow Metab 33: 396-406, 2013.

29. Gage FH: Mammalian neural stem cells. Science 287: 1433-1438, 2000.

30. Eriksson PS, Perfilieva E, Björk-Eriksson T, Alborn AM, Nordborg C, Peterson DA and Gage FH: Neurogenesis in the adult human hippocampus. Nat Med 4: 1313-1317, 1998.

31. Bayer SA, Yackel JW and Puri PS: Neurons in the rat dentate gyrus granular layer substantially increase during juvenile and adult life. Science 216: 890-892, 1982.
32. Temple S and Alvarez-Buylla A: Stem cells in the adult mammalian central nervous system. Curr Opin Neurobiol 9: 135-141, 1999.

33. Kuhn HG and Svendsen CN: Origins, functions, and potential of adult neural stem cells. BioEssays 21: 625-630, 1999.

34. Wang B, Feng G, Tang C, Wang L, Cheng H, Zhang Y, Ma J, Shi M and Zhao G: Ginsenoside Rd maintains adult neural stem cell proliferation during lead-impaired neurogenesis. Neurol Sci 34: 1181-1188, 2013.

35. Lee SH, Kim MH and Han HJ: Arachidonic acid potentiates hypoxia-induced VEGF expression in mouse embryonic stem cells: Involvement of Notch, Wnt, and HIF-lalpha. Am J Physiol Cell Physiol 297: C207-C216, 2009.

36. Land SC and Tee AR: Hypoxia-inducible factor 1alpha is regulated by the mammalian target of rapamycin (mTOR) via an mTOR signaling motif. J Biol Chem 282: 20534-20543, 2007.

37. Tokuda H, Adachi S, Matsushima-Nishiwaki R, Kato K, Natsume H, Otsuka T and Kozawa O: Enhancement of basic fibroblast growth factor-stimulated VEGF synthesis by Wnt3a in osteoblasts. Int J Mol Med 27: 859-864, 2011.

38. Ferrara N: Vascular endothelial growth factor: Basic science and clinical progress. Endocr Rev 25: 581-611, 2004.

39. Tang B, Wang D, Li M, Wu Q, Yang Q, Shi W and Chen C: An in vivo study of hypoxia-inducible factor-1 $\alpha$ signaling in ginsenoside $\mathrm{Rg} 1$-mediated brain repair after hypoxia/ischemia brain injury. Pediatr Res 81: 120-126, 2017.

40. Si YC, Zhang JP, Xie CE, Zhang LJ and Jiang XN: Effects of Panax notoginseng saponins on proliferation and differentiation of rat hippocampal neural stem cells. Am J Chin Med 39: 999-1013, 2011.

41. Jori FP, Galderisi U, Piegari E, Cipollaro M, Cascino A, Peluso G, Cotrufo R, Giordano A and Melone MA: EGF-responsive rat neural stem cells: Molecular follow-up of neuron and astrocyte differentiation in vitro. J Cell Physiol 195: 220-233, 2003.

42. Meng XT, Li C, Dong ZY, Liu JM, Li W, Liu Y, Xue H and Chen D: Co-transplantation of bFGF-expressing amniotic epithelial cells and neural stem cells promotes functional recovery in spinal cord-injured rats. Cell Biol Int 32: 1546-1558, 2008.

This work is licensed under a Creative Commons Attribution-NonCommercial-NoDerivatives 4.0 International (CC BY-NC-ND 4.0) License. 